\title{
ICNMM2008-62222
}

\section{AN EXTENSION TO THE NAVIER-STOKES-FOURIER EQUATIONS BY CONSIDERING MOLECULAR COLLISIONS WITH BOUNDARIES}

\author{
Erik J. Arlemark; S. Kokou Dadzie and Jason M. Reese \\ Department of Mechanical Engineering \\ University of Strathclyde \\ Glasgow, G1 1XJ \\ United Kingdom \\ Email: erik.arlemark@strath.ac.uk / kokou.dadzie@strath.ac.uk / \\ jason.reese@strath.ac.uk
}

\begin{abstract}
In this paper we propose a model for micro gas flows consisting of the Navier-Stokes-Fourier equations (NSF) extended by a description of molecular collisions with solid boundaries and discontinuous velocity slip and temperature jump boundary conditions. By considering the molecular collisions with the solid boundaries in gas flows we capture some of the near wall effects that the conventional NSF with linear stress/strain-rate and heat-flux/temperature-gradient relationships seem to be unable to describe.

The model that we propose incorporates the molecular collisions with solid boundaries as an extension to the conventional definition of the average travelling distance of molecules before experiencing intermolecular collisions (the mean free path). By considering both of these types of collisions we obtain an effective mean free path expression, which varies with distance to surfaces. The effective mean free path is proposed to be used to obtain new definitions of effective viscosity and effective thermal conductivity, which will extend the applicability of NSF equations to higher Knudsen numbers.

We show results of simple flow cases that are solved using this extended NSF model and discuss limitations to the model due to various assumptions. We also mention interesting ideas for further development of the model based on a more detailed gas description.
\end{abstract}

\footnotetext{
* Address all correspondence to this author.
}

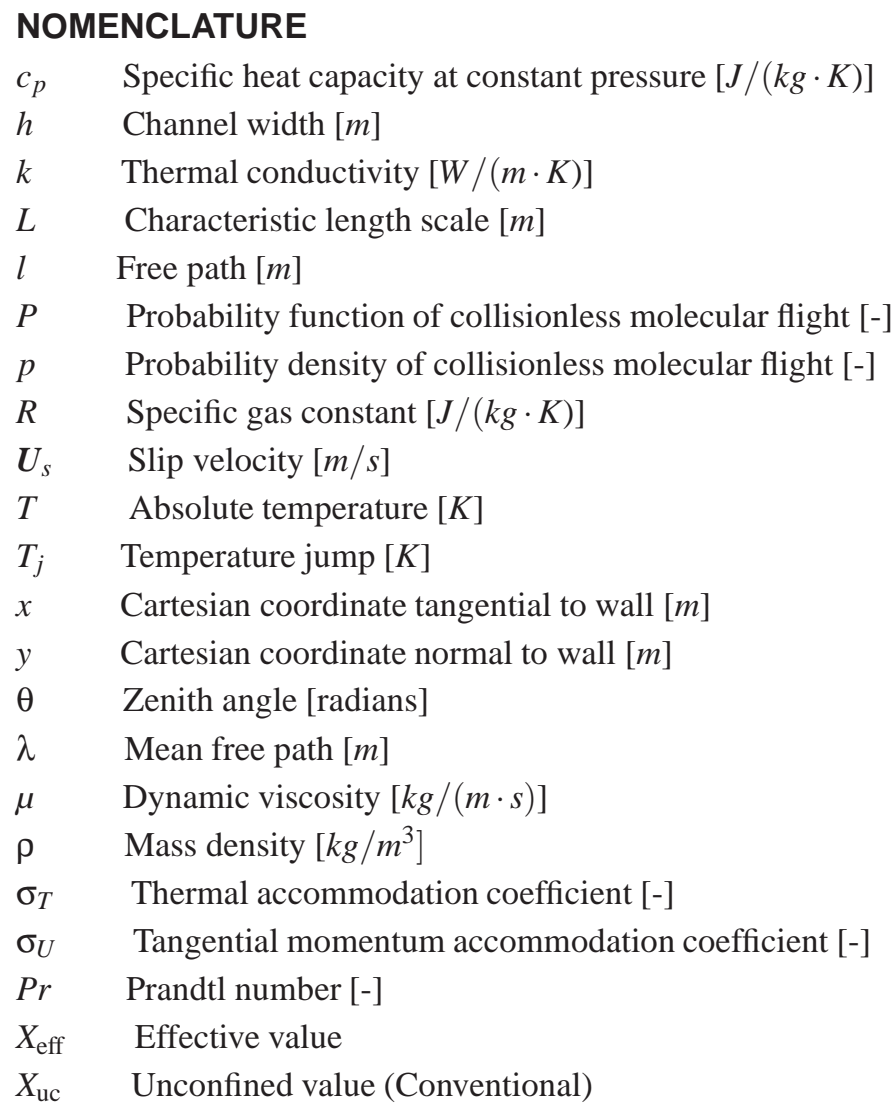

NOMENCLATURE 


\section{INTRODUCTION}

As our interest in micro-gas-flow applications is growing with improved manufacturing capabilities it is also realised that conventional flow modelling methods, consisting of the NavierStokes-Fourier (NSF) equations with no-velocity-slip and notemperature-jump boundary conditions, fail for such cases. This is because micro-gas-flows differ from macro-gas-flows with respect to the relatively large ratio of the confining boundary surface area to the volume of the confined gas, which means that certain surface effects must be taken into account. These surface effects considerably influence the flow in the near-wall region, the Knudsen-layer, which is about one to two mean free paths (MFP) wide. The MFP-expression referred to in this paper is derived for molecules with extended potentials, and in terms of dynamic viscosity [1]:

$$
\lambda_{\mathrm{uc}}=\frac{\mu_{\mathrm{uc}}}{\rho} \sqrt{\frac{\pi}{2 R T}} .
$$

The key parameter, the Knudsen number $(K n)$, is then:

$$
K n=\frac{\lambda_{\text {uc }}}{L},
$$

where we use the channel width, $h$, as the value of $L$ since only planar wall cases are evaluated in this paper.

Modelling of surface effects in the Knudsen-layer should ideally be performed using detailed kinetic theory. However an approximate extension to the NSF model would be less timeconsuming and less demanding of computational capacity.

Surface effects for rarefied gases up to $\mathrm{Kn} \approx 0.1$ can be modelled by applying the boundary conditions of velocity-slip and temperature-jump. Above $\mathrm{Kn} \approx 0.1$ non-linear relationships for shear-stress and heat-flux also need to be applied to NSF to account for the surface effects, as the Knudsen-layer covers an even larger part of the flow-domain.

The model in this paper extends the validity of NSF beyond $\mathrm{Kn} \approx 0.1$ by incorporating non-linear constitutive relationships together with first order boundary conditions, which is achieved by describing molecular behaviour in the near-wall regions. The non-linear constitutive relationships are derived by using new definitions of geometry-dependent viscosity and thermal conductivity which are based on our model of a shortening of the MFP due to wall collisions. This proposed definition of the MFP is geometry-dependent and referred to as the effective mean free path, $\lambda_{\text {eff }}$.

\section{DISCONTINUOUS BOUNDARY CONDITIONS}

Traditionally, according to Gad-el-Hak [2], the reasoning is made that there cannot exist discontinuities at the gas-solid interface. This reasoning is based on the fact that the gas then would have infinite gradients of velocity and temperature, which would correspond to the unphysical effect of infinite viscous stresses and heat fluxes.

For micro-gas-flows however a state of non-equilibrium exists in the Knudsen-layers. In addition it is now well accepted that the use of slip velocity and temperature jump improve the description of micro-gas-flows [2,3].

The velocity-slip that we adopt in this paper, is the slipexpression by Maxwell [4] for planar walls:

$$
\boldsymbol{U}_{s}=\left.\frac{2-\sigma_{U}}{\sigma_{U}} \lambda\left(\frac{\partial \boldsymbol{U}}{\partial y}\right)\right|_{w}+\left.\frac{3}{4} \frac{\mu}{\rho T}\left(\frac{\partial T}{\partial x}\right)\right|_{w}
$$

where the subscript $w$ denotes values at the wall. Conventionally, the velocity profile is extrapolated over the Knudsen-layer from flow values taken one MFP away from the wall. The tangential momentum accommodation coefficient, $\sigma_{U}$, is defined for tangential momentum exchange of gas molecules with surfaces. Theoretically this quantity takes a value of 0 for purely specular reflections and 1 for purely diffusive reflections. Values for $\sigma_{U}$ have been inferred from experimental comparisons by Arkilic [5] and have been found to be in the range of 0.75 to 0.85 for argon, nitrogen and carbon dioxide confined by silicon surfaces.

To illustrate the impact of Maxwell's slip-velocity, with only diffusive reflections, on the one-dimensional isothermal NSF equations we present results of a Couette flow. For this case the NSF equations reduce to the governing equation:

$$
\frac{\partial}{\partial y}\left(\mu_{\mathrm{uc}} \frac{\partial U_{x}}{\partial y}\right)=0
$$

where it is assumed that the flow is only travelling in the $x$ direction and it is non-uniform only in the $y$-direction i.e. $U_{x}=$ $U_{x}(y)$.

The solution method by Vincenti [6] for solving Eqn. (4) using the first term of the slip boundary condition of Eqn. (3) then yields:

$$
\frac{U_{x}}{U_{w}}=\frac{y}{h}\left(\frac{1}{1+2 K n}\right)=\frac{y}{h}\left(\frac{1}{1+2 \lambda_{\mathrm{uc}} / h}\right),
$$

where $U_{w}$ is the wall velocity and $y$ has its reference origin in the middle of the channel. The normalised velocity profile is shown in Fig. 1 together with the more reliable results of Sone et al. [7] attained by the linearised Boltzmann equation.

The NSF model using Maxwell's slip velocity boundary condition shows adequate results in comparison to the result of 


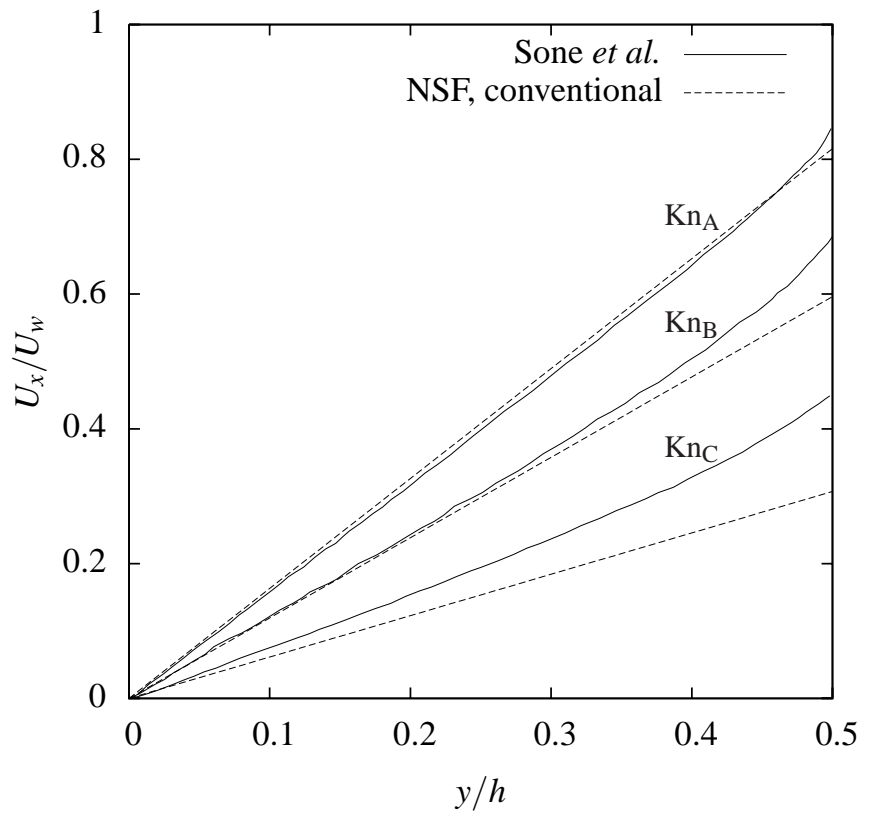

Figure 1. COUETTE FLOW SOLVED USING CONVENTIONAL NSF COMPARED WITH THE LINEARISED BOLTZMANN EQUATION SOLVED BY Sone et al. [7] FOR $\mathrm{Kn}_{\mathrm{A}}=0.113, \quad \mathrm{~K} \mathrm{n}_{\mathrm{B}}=0.339$ AND $\mathrm{Kn}_{\mathrm{C}}=1.128$. HERE $y$ IS ZERO IN THE CHANNEL CENTRE.

Sone et al. [7] for $\mathrm{Kn}_{\mathrm{A}}=0.113$. For the cases at higher $\mathrm{Kn}$ it is seen that the NSF velocity-profiles deviate significantly from the results of Sone et al. [7].

In the next section we outline how a near-wall molecular description may be adopted into the NSF equations in order to extend their prediction capabilities to higher Kn.

\section{NON-LINEAR CONSTITUTIVE RELATIONSHIPS}

The theory of our proposed non-linear constitutive relationships is based on the view of viscosity as a momentum exchange between molecules, which is dependent on the time between molecular collisions and therefore also on the MFP of molecular flights. The relation between the MFP, that is not influenced by anything but other gas molecules, and the viscosity is established in Eqn. (1). However, in micro-gas-flows there is a nonnegligible shortening of the free path due to molecular collisions with the unyielding walls, which makes it reasonable to use an effective-MFP instead of the unconfined-MFP. The corresponding effective viscosity is expressed as:

$$
\mu_{\text {eff }}=\rho \frac{\lambda_{\text {eff }}}{\sqrt{\pi / 2 R T}} .
$$

Through this expression and the definition of the Prandtl number, $\operatorname{Pr}=c_{p} \mu / k$, an effective thermal conductivity, $k_{\mathrm{eff}}$, can be attained by replacing $\mu$ and $k$ by their corresponding new effective definitions as follows:

$$
k_{\mathrm{eff}}=\frac{c_{p} \mu_{\mathrm{eff}}}{\operatorname{Pr}}
$$

Next we will present how the dependent quantity of $\lambda_{\text {eff }}$ can be defined and state the assumptions involved.

\section{EFFECTIVE MEAN FREE PATH}

The method of using transport parameters which are influenced by an effective MFP is traced back to Stops [8]. Stops proposes an effective-MFP model, $\lambda_{\text {eff(S) }}$, which is derived based on a probability density,

$$
p(r)=\frac{1}{\lambda_{\mathrm{uc}}} \exp \left(-\frac{r}{\lambda_{\mathrm{uc}}}\right),
$$

which describes the distribution of the free path in terms of the flight length $r$. The value of the MFP can then be obtained by integrating $r \times p(r)$, with respect to $r$, from zero to infinity.

The $\lambda_{\text {eff(S) }}$ model is derived by shortening the upper integrational limit of $r$ from infinity to the distance to the confining wall as well as using solid-angle-analysis. This is done while taking into consideration that the integrational path of $r$ is equally probable of being distributed in any zenith angle, $\theta$, between the molecule and the wall. This integration yields the following $\lambda_{\text {eff(S) }}$ expression for molecules in a planar wall confinement:

$$
\begin{aligned}
\lambda_{\mathrm{eff}(\mathrm{S})}= & \frac{\lambda_{\mathrm{uc}}}{2}\left\{1+\left(\frac{y}{\lambda_{\mathrm{uc}}}-1\right) \exp \left(-\frac{y}{\lambda_{\mathrm{uc}}}\right)\right. \\
& \left.-\left(\frac{y}{\lambda_{\mathrm{uc}}}\right)^{2} E i\left(\frac{y}{\lambda_{\mathrm{uc}}}\right)\right\}+\frac{\lambda_{\mathrm{uc}}}{2},
\end{aligned}
$$

where $y$ is the normal distance to a wall and $E i$ is the exponential integral function defined as:

$$
E i(x)=\int_{1}^{\infty} t^{-1} \exp (-x t) d t
$$

The last term of Eqn. (9) assumes that molecules are unconfined when travelling in the direction opposite to the wall. If the confining walls are close enough for a considerable amount 
of Knudsen-layer-overlap to occur, $\lambda_{\mathrm{eff}(\mathrm{S})}$ is expressed as [9]:

$$
\begin{aligned}
\lambda_{\mathrm{eff}(\mathrm{S})}= & \frac{\lambda_{\mathrm{uc}}}{2}\left\{2+\left(\frac{y}{\lambda_{\mathrm{uc}}}-1\right) \exp \left(-\frac{y}{\lambda_{\mathrm{uc}}}\right)-\left(\frac{y}{\lambda_{\mathrm{uc}}}\right)^{2} E i\left(\frac{y}{\lambda_{\mathrm{uc}}}\right)\right. \\
& +\left(\frac{h-y}{\lambda_{\mathrm{uc}}}-1\right) \exp \left(\frac{y-h}{\lambda_{\mathrm{uc}}}\right) \\
& \left.-\left(\frac{h-y}{\lambda_{\mathrm{uc}}}\right)^{2} E i\left(\frac{h-y}{\lambda_{\mathrm{uc}}}\right)\right\}
\end{aligned}
$$

In the next section an expression similar to $\lambda_{\text {eff(S) }}$ is derived that is not dependent on the Ei-function and may therefore be easier to implement for flow case calculations.

\section{Probability-function based effective-MFP expression}

We will use the integrated form of the density function $p(r)$ referred to as the probability function,

$$
P(r)=\int p(r) d r=C-\exp \left(-\frac{r}{\lambda_{\mathrm{uc}}}\right) .
$$

This function describes the probability for a molecule to travel a distance $r+d r$ between two successive collisions, where the integration constant, $C$, is set to one for the probability to range from zero to one.

This model is derived for a planar-wall-configuration, as shown in Fig. 2, where the molecules can either travel in the negative $y$-direction, where $r=y / \cos (\theta)$, or travel in the positive $y$-direction, where $r^{\prime}=(h-y) / \cos \left(\theta^{\prime}\right)$ if the channel width is $h$.

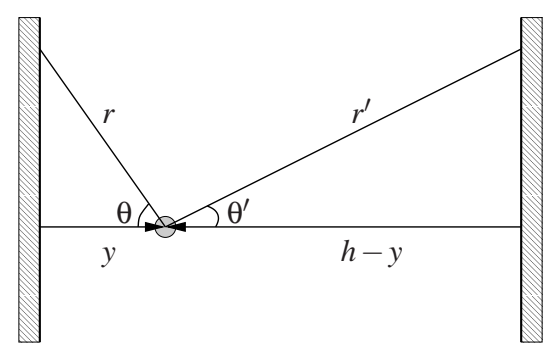

Figure 2. A MOLECULE CONFINED BETWEEN TWO PLANAR WALLS WITH SPACING $h$. THE PRIME SUPERSCRIPTS DENOTE QUANTITIES CORRESPONDING TO THE RIGHT WALL.

In this description a molecule has an equal possibility to travel in either positive or negative $y$-direction. The distribution of the free path, $l$, may then be described by using $P$ as a weighting function as follows:

$$
\begin{aligned}
l & =\lambda_{\mathrm{uc}} \times \frac{1}{2}\left[P(r)+P\left(r^{\prime}\right)\right] \\
& =\lambda_{\mathrm{uc}} \times\left\{1-\frac{1}{2}\left[\exp \left(-\frac{r}{\lambda_{\mathrm{uc}}}\right)+\exp \left(-\frac{r^{\prime}}{\lambda_{\mathrm{uc}}}\right)\right]\right\} .
\end{aligned}
$$

If the configuration only consists of one wall or the right wall is at a considerable distance i.e. $(h>>y)$ this expression reduces to:

$$
l=\lambda_{\mathrm{uc}} \times P(r)=\lambda_{\mathrm{uc}} \times\left\{1-\frac{1}{2} \exp \left(-\frac{r}{\lambda_{\mathrm{uc}}}\right)\right\}
$$

A mean free path depending on the molecule's distance to the wall is obtained by averaging the free path with respect to $\theta$ in the range of $[0, \pi / 2]$. This is done by using the mean integral theorem,

$$
\langle X(\theta)\rangle=\frac{1}{(\pi / 2-0)} \int_{0}^{\pi / 2} X(\theta) d \theta
$$

on the $\theta$ dependent exponential-part of the $l$-expressions, where the integrational domain is illustrated in Fig. 3. Using this averaging-method over the travelling direction is the main difference in our model compared to the Stops' method.



Figure 3. A MOLECULE AT A DISTANCE, $y$, FROM A PLANAR WALL. CYLINDRICAL COORDINATES $(y, y \tan (\theta))$ INDICATES POSSIBLE TRAVELLING DIRECTIONS FOR A MOLECULE TRAVELLING IN THE NEGATIVE $y$-DIRECTION.

The mean integral theorem is evaluated by Simpson's numerical integration with 4 subintervals, which for the case with 
two walls yields:

$$
\begin{aligned}
\lambda_{\mathrm{eff}}= & \lambda_{\mathrm{uc}}-\frac{\lambda_{\mathrm{uc}}}{22}\left[\exp \left(-\frac{y}{\lambda_{\mathrm{uc}}}\right)+\exp \left(-\frac{h-y}{\lambda_{\mathrm{uc}}}\right)\right. \\
& +4 \sum_{i=1}^{2} \exp \left(-\frac{y}{\cos ((2 i-1) \pi / 8) \lambda_{\mathrm{uc}}}\right) \\
& +4 \sum_{i=1}^{2} \exp \left(-\frac{h-y}{\cos ((2 i-1) \pi / 8) \lambda_{\mathrm{uc}}}\right) \\
& +2 \exp \left(-\frac{y}{\cos (\pi / 4) \lambda_{\mathrm{uc}}}\right) \\
& \left.+2 \exp \left(-\frac{h-y}{\cos (\pi / 4) \lambda_{\mathrm{uc}}}\right)\right]
\end{aligned}
$$

For the one-wall case, Eqn. (16) reduces to:

$$
\begin{aligned}
\lambda_{\mathrm{eff}}= & \lambda_{\mathrm{uc}}-\frac{\lambda_{\mathrm{uc}}}{22}\left[\exp \left(-\frac{y}{\lambda_{\mathrm{uc}}}\right)\right. \\
& +4 \sum_{i=1}^{2} \exp \left(-\frac{y}{\cos ((2 i-1) \pi / 8) \lambda_{\mathrm{uc}}}\right) \\
& \left.+2 \exp \left(-\frac{y}{\cos (\pi / 4) \lambda_{\mathrm{uc}}}\right)\right]
\end{aligned}
$$

The result of the effective-MFP-model, for one wall given in Eqn. (17), is presented in Fig. 4. The present model shows that the model fulfilles the basic requirements of:

$$
\lambda_{\mathrm{eff}}(0)=\frac{\lambda_{\mathrm{uc}}}{2}, \quad \lambda_{\mathrm{eff}}(\infty)=\lambda_{\mathrm{uc}}
$$

The requirement at the wall can be realised by considering the average of the equal probability of a molecule travelling in the direction towards the confining wall, yielding a contribution of 0 , as of travelling into the bulk of the flow, yielding a contribution of $\lambda_{\text {uc }}$. The requirement for molecules far away from the wall states that the effective-MFP should approach the conventional and unconfined-value.

The results of the effective-MFP-model for two walls, given in Eqn. (16), is presented in Fig. 5 for the two $K n=\lambda_{\text {uc }} / h=0.25$ and 1 . As seen in the results of $K n=0.25$, both models are quite similar and have a near wall value that fulfills the first basic requirement of Eqn. (18) and they almost approach the unconfined value in the bulk. For $K n=1$ the models show slightly different results. For this case the $\lambda_{\text {eff }}$-models has near wall values that are lower than $\lambda_{\mathrm{uc}} / 2$ and the bulk values are considerably lower than $\lambda_{\text {uc }}$, which is consistent with that the effective-MFP-profiles are relatively flat compared to the $0.25 \mathrm{Kn}$ case.

In the next section we will present simple test cases using the present $\lambda_{\text {eff }}$-model.



Figure 4. COMPARISON OF DIFFERENT $\lambda_{\text {eff-MODELS THAT VARY }}$ WITH WALL DISTANCE, $y$.

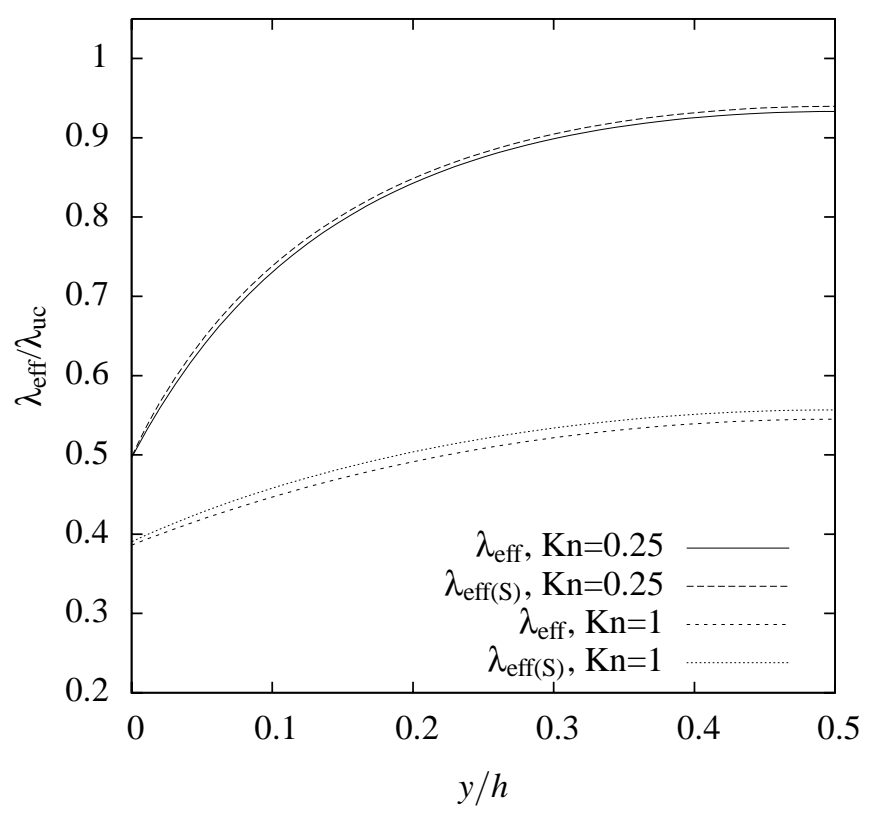

Figure 5. COMPARISON OF DIFFERENT $\lambda_{\text {eff-MODELS THAT VARY }}$ WITH WALL DISTANCE, $y$. 


\section{TEST CASE RESULTS}

In this section the isothermal Couette and Poiseuille cases are solved analytically with NSF using a $\mu_{\text {eff }}$ derived from the

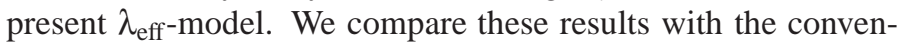
tional NSF and solutions to the linearised Boltzmann equation by Ohwada et al. [10] and Sone et al. [7]. The Poiseuille flow solution by Ohwada et al. gave good results on the prediction of mass flow rate in microchannel when compared to experiments for Kn up to 0.5 .

\section{Couette flow}

Here we study the same Couette case as above, but using an effective viscosity from our effective-MFP model instead of the corresponding unconfined value. Since the flow only varies in the $y$ direction the governing equation is given by:

$$
\frac{\partial}{\partial y}\left(\mu_{\mathrm{eff}} \frac{\partial U_{x}}{\partial y}\right)=0
$$

where $y$ has its reference origin in the middle of the channel. The two velocity boundary conditions are:

$$
U_{x}(h / 2)=\left.\frac{2-\sigma_{U}}{\sigma_{U}} \lambda_{\mathrm{eff}} \frac{\partial U_{x}}{\partial y}\right|_{w} \quad \text { and } \quad U_{x}(0)=0
$$

where $\sigma_{U}=1$. The velocity profile can then be expressed by:

$$
\frac{U_{x}}{U_{w}}=\frac{F(y)-F(0)}{F(h / 2)+\lambda_{\mathrm{eff}}(h / 2) f(h / 2)-F(0)},
$$

where

$$
f(y)=\frac{1}{\mu_{\mathrm{eff}}(y)} \quad \text { and } \quad F(y)=\int \frac{1}{\mu_{\mathrm{eff}}(y)} d y .
$$

The results of Eqn. (21) are shown in Fig. 6. Here it is seen that the extended-NSF-model shows a slightly lower velocityprofile but with a similar curvature to the results of Sone et al. [7] for the cases of $\mathrm{Kn}_{\mathrm{A}}$ and $\mathrm{Kn}_{\mathrm{B}}$. In the case of $\mathrm{Kn}_{\mathrm{C}}$ the extendedNSF-model shows a slightly higher velocity-profile but a similar curvature relative to the results by Sone et al. [7].



Figure 6. COUETTE FLOW RESULTS USING CONVENTIONAL NSF

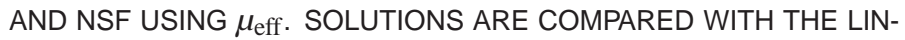
EARISED BOLTZMANN SOLUTION OF Sone et al. [7] FOR $\mathrm{Kn}_{\mathrm{A}}=0.113$, $\mathrm{Kn}_{\mathrm{B}}=0.339$ AND $\mathrm{Kn}_{\mathrm{C}}=1.128$. HERE $y=0$ IS THE CHANNEL CENTRE.

\section{Poiseuille flow}

Now we study the Poiseuille flow case represented by the following governing equation:

$$
\frac{\partial}{\partial y}\left(\mu_{\mathrm{eff}} \frac{\partial U_{x}}{\partial y}\right)=\frac{\partial p}{\partial x}
$$

where $y$ has its reference origin in the middle of the channel and $x$ at the inlet. The two velocity boundary conditions are:

$$
U_{x}(h / 2)=\left.\frac{2-\sigma_{U}}{\sigma_{U}} \lambda_{\text {eff }} \frac{\partial U_{x}}{\partial y}\right|_{w} \quad \text { and }\left.\quad \frac{\partial U_{x}}{\partial y}\right|_{y=0}=0
$$

where $\sigma_{U}=1$. The velocity profile is then described by:

$$
\frac{U_{x}}{U_{0}}=\frac{1}{U_{0}} \frac{\partial p}{\partial x}\left[G(y)-\frac{\lambda_{\mathrm{eff}}(h / 2)}{\mu_{\mathrm{eff}}(h / 2)} \frac{h}{2}-G(h / 2)\right],
$$

where

$$
G(y)=\int \frac{y}{\mu_{\mathrm{eff}}(y)} d y \quad \text { and } \quad U_{0}=-\frac{h^{2}}{8 \mu_{\mathrm{uc}}} \frac{\partial p}{\partial x} .
$$


The velocity profiles obtained from Eqn. (25) are represented in Fig. 7 together with results obtained by Ohwada et al. [10] using the linearised Boltzmann equation. We also compare our present results with the conventional NSF using the conventional boundary conditions of Eqn. (23) and Eqn. (24) with the effective values replaced by unconfined values. The solution of the conventional NSF is given by:

$$
\frac{U_{x}}{U_{0}}=1-4 y / h+4 K n=1-4 y / h+4 \lambda_{\mathrm{uc}} / h
$$

where the solution method is from [11].

In Fig. 7 we show the results by Ohwada et al. [10], the NSF using $\mu_{\text {eff }}$ and the conventual NSF. It is seen that the conventional NSF and NSF using $\mu_{\mathrm{eff}}$ deviates slightly from the results by Ohwada et al. [10] for $\mathrm{Kn}_{\mathrm{A}}=0.113$. For $\mathrm{Kn}_{\mathrm{B}}=0.451$ the conventional NSF deviates significantly from the results by Ohwada et al. [10] while NSF using $\mu_{\text {eff }}$ show better results. None of the NSF models describe the results of Ohwada et al. [10] well for $\mathrm{Kn}_{\mathrm{C}}=0.903$.

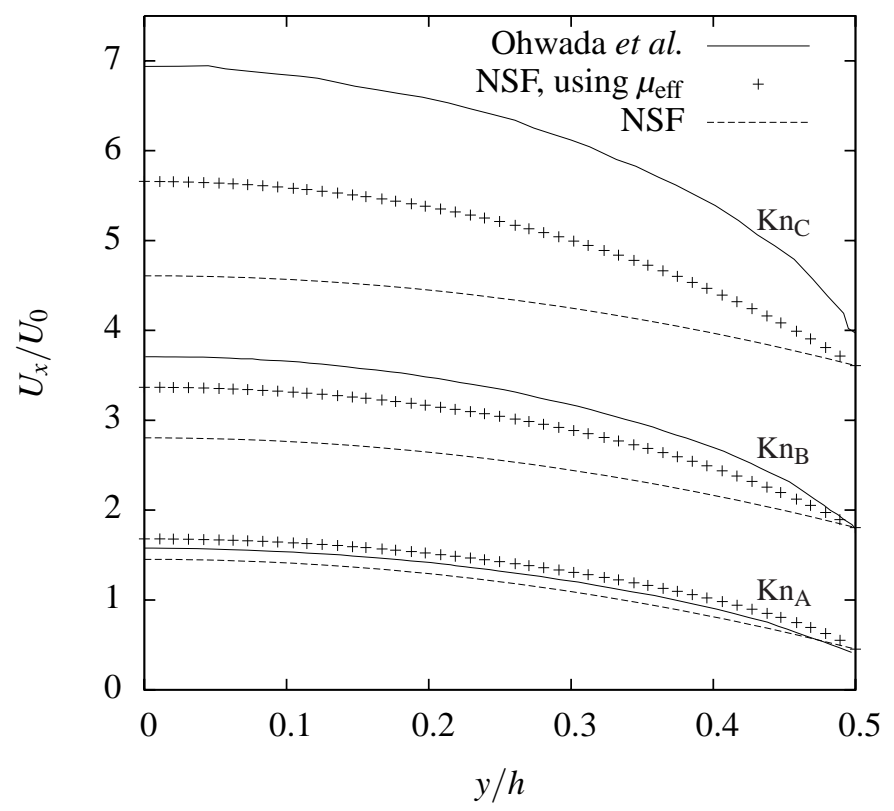

Figure 7. POISEUILLE FLOW RESULTS USING CONVENTIONAL

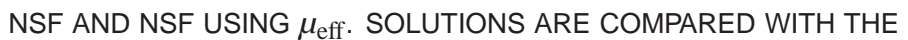
LINEARISED BOLTZMANN EQUATION SOLVED BY Ohwada et al. [10] FOR $\mathrm{Kn}_{\mathrm{A}}=0.113, \mathrm{Kn}_{\mathrm{B}}=0.451$ AND $\mathrm{Kn}_{\mathrm{C}}=0.903$. HERE $y=0$ IS THE CHANNEL CENTRE.

\section{CONCLUSIONS}

The need to justify which $\lambda_{\text {eff }}$-value should be used in the velocity-slip condition still remains. However, it is seen that this extension to NFS by using an effective-viscosity manages to capture the shape of the velocity profile of the comparison-data and the near wall velocity values up to $\mathrm{Kn} \approx 0.5$. We therefore propose further investigation of this extended-NSF-model for more complex cases involving heat transfer and more difficult geometries such as cylindrical Couette flows. It is also of interest to investigate the the ability to model rarefied gas flows above Kn $\approx 0.5$ using second order boundary conditions.

\section{Possible improvements to the effective-MFP-model}

We consider that our effective-MFP-model reproduces the results of Stops' effective-MFP-model quite well, which is expected because they are both derived based on a similar description. Interesting continuation of this work would be to extend the validity of the effective-MFP to curved surfaces, although curvature that is small in comparison with the width of the Knudsenlayer will have a negligible impact on the effective-MFP expression. Another interesting area of continuation of this work is to include more physical consistency to the model such as to distinguish between intermolecular collisions and molecular collisions with solid boundaries. This distinction may be easier to implement in the present model than in Stops' model.

\section{ACKNOWLEDGMENT}

This research is funded in the UK by the Engineering and Physical Sciences Research Council under grant no. EP/D007488/1.

\section{REFERENCES}

[1] Cercignani, C., 2000. Rarefied Gas Dynamics. Cambridge University Press.

[2] Gad-el-Hak, M., 1999. "Fluid mechanics of microdevicesthe Freeman Scholar lecture". Journal of Fluids Engineering, Transactions of the ASME, 121(1), pp. 5-33.

[3] Arkilic, E., Schmidt, M., and Breuer, K., 1997. "Gaseous slip flow in long microchannels". Journal of Microelectromechanical Systems, 6(2), pp. 167-178.

[4] Maxwell, J., 1879. "On stresses in rarified gases arising from inequalities of temperature". Philosophical Transactions of the Royal Society of London, 170, pp. 231-256.

[5] Arkilic, E., Breuer, K., and Schmidt, M., 2001. "Mass flow and tangential momentum accomodation in silicon micromachined channels". Journal of Fluid Mechanics, 437, pp. 29-43.

[6] Vincenti, W., and Kruger, C., 2002. Introduction to Physical Gas Dynamics. Krieger. 
[7] Sone, Y., Takata, S., and Ohwada, T., 1990. "Numerical analysis of the plane Couette flow of a rarefied gas on the basis of the linearized Boltzmann equation for hardsphere molecules". European Journal of Mechanics, B, 9(3), pp. 273-288.

[8] Stops, D. W., 1970. "The mean free path of gas molecules in the transition regime". Journal of Physics D, 3(5), pp. 685-696.

[9] Guo, Z. L., Shi, B. C., and Zheng, C. G., 2007. "An extended Navier-Stokes formulation for gas flows in the Knudsen layer near a wall”. Europhysics Letters, 80(2), pp. 24001-24006.

[10] Ohwada, T., Sone, Y., and Aoki, K., 1989. "Numerical analysis of the poiseuille and thermal transpiration flows between two parallel plates on the basis of the boltzmann equation for hard-sphere molecules". Physics of Fluids, A, 1(12), pp. 2042-2049.

[11] Kandlikar, S., Garimella, S., Li, D., Colin, S., and King, M. R., 2005. Heat Transfer and Fluid Flow in Minichannels and Microchannels. Elsevier. 